\title{
Spin-liquid and magnetic phases in the anisotropic triangular lattice: The case of $\kappa-(\mathrm{ET})_{2} \mathrm{X}$
}

\author{
Luca F. Tocchio, ${ }^{1}$ Alberto Parola, ${ }^{2}$ Claudius Gros, ${ }^{1}$ and Federico Becca ${ }^{3}$ \\ ${ }^{1}$ Institute for Theoretical Physics, Goethe-University Frankfurt, Max-von-Laue-Straße 1, D-60438 Frankfurt am Main, Germany \\ ${ }^{2}$ Dipartimento di Fisica e Matematica, Università dell'Insubria, Via Valleggio 11, I-22100 Como, Italy \\ ${ }^{3}$ CNR-INFM-Democritos National Simulation Centre and International School for Advanced Studies (SISSA), \\ Via Beirut 2, I-34151 Trieste, Italy \\ (Received 24 July 2009; published 26 August 2009)
}

\begin{abstract}
The two-dimensional Hubbard model on the anisotropic triangular lattice, with two different hopping amplitudes $t$ and $t^{\prime}$, is relevant to describe the low-energy physics of $\kappa-(\mathrm{ET})_{2} \mathrm{X}$, a family of organic salts. The ground-state properties of this model are studied by using Monte Carlo techniques, on the basis of a recent definition of backflow correlations for strongly correlated lattice systems. The results show that there is no magnetic order for reasonably large values of the electron-electron interaction $U$ and frustrating ratio $t^{\prime} / t$ $=0.85$, suitable to describe the nonmagnetic compound with $\mathrm{X}=\mathrm{Cu}_{2}(\mathrm{CN})_{3}$. On the contrary, Néel order takes place for weaker frustrations, i.e., $t^{\prime} / t \sim 0.4-0.6$, suitable for materials with $\mathrm{X}=\mathrm{Cu}_{2}(\mathrm{SCN})_{2}, \mathrm{Cu}\left[\mathrm{N}(\mathrm{CN})_{2}\right] \mathrm{Cl}$, or $\mathrm{Cu}\left[\mathrm{N}(\mathrm{CN})_{2}\right] \mathrm{Br}$.
\end{abstract}

DOI: 10.1103/PhysRevB.80.064419

PACS number(s): 71.10.Fd, 71.27.+a, 71.30.+h, 75.10.-b

\section{INTRODUCTION}

Organic charge-transfer salts show a wide variety of quantum phases and represent prominent examples to study correlation effects in low-dimensional systems. The most celebrated case is given by the TTF-TCNQ salt that has been primarily regarded as a prototype for testing theories of onedimensional conductors. ${ }^{1}$ Organic salts may also form crystals in two and three dimensions, and, in this respect, an increasing attention has been devoted to a particular family denoted by $\kappa-(\mathrm{ET})_{2} \mathrm{X}$, whose building block is the so-called BEDT-TTF (or ET) molecule and $\mathrm{X}$ is a monovalent anion. ${ }^{2}$ Here, strongly dimerized ET molecules are arranged in a two-dimensional triangular lattice. Each dimer has a charge state with one hole and therefore the conducting band is half filled. A sizable effective-Coulomb repulsion is felt by two holes on the same dimer. A huge variety of phases have been found (by varying temperature, pressure, or the nature of the anion $\mathrm{X}$ ), ranging from correlated (bad) metals with superconductivity at low temperatures, to Mott insulators with magnetic order. ${ }^{3-6}$ Interestingly, by acting with hydrostatic pressure, metal-insulator transitions have been observed, ${ }^{7,8}$ with the remarkable possibility to stabilize a nonmagnetic Mott insulating phase in $\kappa-(\mathrm{ET})_{2} \mathrm{Cu}_{2}(\mathrm{CN})_{3} \cdot{ }^{9}$ In this material, there is no evidence of magnetic order down to $T \simeq 30 \mathrm{mK}$, which is four orders of magnitude lower than the estimate of the superexchange coupling $J \simeq 250 \mathrm{~K}$.

It has been argued that $\kappa$-(ET) ${ }_{2} \mathrm{X}$ compounds can be described by a single-band Hubbard model on the anisotropic triangular lattice, ${ }^{10}$ where chains described by an hopping $t^{\prime}$ are coupled together with zigzag hoppings $t$, see Fig. 1. An on-site repulsive term $U$ is also present in the Hamiltonian. However, a realistic estimate of these microscopic parameters is not exempt from complications. Indeed, the values obtained some time ago by extended Hückel band-structure calculations ${ }^{11}$ have been put in doubt by two recent $a b$ initio calculations, based upon local-density approximation and generalized gradient approximation. ${ }^{12,13}$ Interestingly, the results suggest that these organic salts are less frustrated than previously assumed and that $t^{\prime} / t$ is smaller than one. Indeed, the frustrating ratio is $t^{\prime} / t \sim 0.8$ for $\kappa-(\mathrm{ET})_{2} \mathrm{Cu}_{2}(\mathrm{CN})_{3}$ and $t^{\prime} / t \sim 0.6$ for $\kappa$ - $(\mathrm{ET})_{2} \mathrm{Cu}_{2}(\mathrm{SCN})_{2} \cdot{ }^{12,13}$ Other materials, with $\mathrm{X}=\mathrm{Cu}\left[\mathrm{N}(\mathrm{CN})_{2}\right] \mathrm{Cl}$ or $\mathrm{Cu}\left[\mathrm{N}(\mathrm{CN})_{2}\right] \mathrm{Br}$, have a substantially smaller frustrating ratio, i.e., $t^{\prime} / t \sim 0.4{ }^{13}$ Unfortunately, an accurate determination of the correlation energy is rather difficult and these two calculations give a considerably different estimation of the Coulomb repulsion, namely, $U / t \sim 12-15$ (Ref. 12) and $U / t \sim 5-7$ (Ref. 13).

Here, we apply our improved Monte Carlo calculations, based upon the recently introduced backflow wave function ${ }^{14}$ in order to analyze the possibility of having a nonmagnetic insulator for large enough frustration and interaction strength. The paper is organized as follows: in Sec. II, we introduce the Hamiltonian; in Sec. III, we describe our variational wave function; in Sec. IV, we present our numerical results and, finally, in Sec. V we draw the conclusions.

\section{MODEL}

We consider the Hubbard model described by

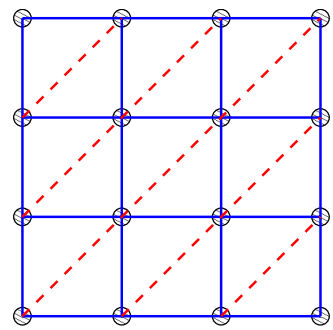

a) b)

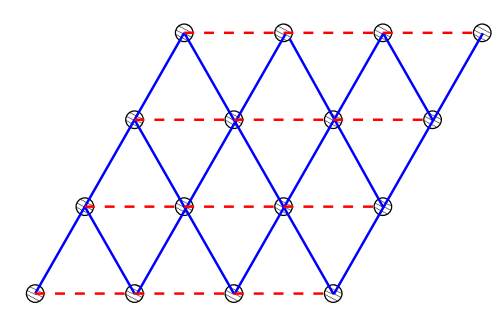

FIG. 1. (Color online) Illustration of the lattice in the square topology (a) used in this work and in the equivalent triangular one. (b) Solid and dashed lines indicate hopping amplitudes $t$ and $t^{\prime}$, respectively. 


$$
\mathcal{H}=-\sum_{i, j, \sigma} t_{i j} c_{i, \sigma}^{\dagger} c_{j, \sigma}+\text { H.c. }+U \sum_{i} n_{i, \uparrow} n_{i, \downarrow},
$$

where $c_{i, \sigma}^{\dagger}\left(c_{i, \sigma}\right)$ creates (destroys) an electron with spin $\sigma$ on site $i, n_{i, \sigma}=c_{i, \sigma}^{\dagger} c_{i, \sigma}, t_{i j}$ is the hopping amplitude and $U$ is the on-site Coulomb repulsion. In this work, we focus our attention on the half-filled case with $N$ electrons on $N$ sites and consider a square lattice with a nearest-neighbor hopping $t$, along the $(1,0)$ and $(0,1)$ directions, and a further nextnearest-neighbor hopping $t^{\prime}$ along $(1,1)$; this choice of the hopping amplitudes is topologically equivalent to the anisotropic triangular lattice, ${ }^{15}$ see Fig. 1. In the last years, an intense effort has been devoted to this problem by use of a large variety of methods, including exact diagonalization, ${ }^{16}$ path-integral renormalization group, ${ }^{17}$ variational Monte Carlo, ${ }^{18-20}$ cluster dynamical mean-field theory, ${ }^{21,22}$ and dual Fermions. ${ }^{23}$ All these methods give rather different outcomes and there are huge discrepancies on the phase boundaries and, most importantly, on the expected nature of the nonmagnetic insulator. The aim of this work is to clarify the ground-state properties for two values of $t^{\prime} / t=0.6$ and 0.85 , relevant for materials with $\mathrm{X}=\mathrm{Cu}_{2}(\mathrm{SCN})_{2}$ and $\mathrm{Cu}_{2}(\mathrm{CN})_{3}$, respectively.

\section{VARIATIONAL APPROACH}

A variational wave function for an insulator with antiferromagnetic (AF) order can be constructed by considering the ground state $|\mathrm{AF}\rangle$ of a mean-field Hamiltonian containing a band contribution and a magnetic term

$$
\mathcal{H}_{\mathrm{AF}}=\sum_{q, \sigma} \epsilon_{q} c_{q, \sigma}^{\dagger} c_{q, \sigma}+\Delta_{\mathrm{AF}} \sum_{i} \mathbf{n}_{i} \cdot \mathbf{S}_{i},
$$

where $\mathbf{n}_{i}$ is a unitary vector that depends upon the lattice site $i$ and $\mathbf{S}_{i}=\left(S_{i}^{x}, S_{i}^{y}, S_{i}^{z}\right)$ is the spin operator. Moreover, $\epsilon_{q}=-2 t\left(\cos q_{x}+\cos q_{y}\right)-2 t_{d} \cos \left(q_{x}+q_{y}\right)$ is a variational band term: $t$ gives the energy scale and $t_{d}$ can be optimized to minimize the variational energy. In order to correctly describe spin-fluctuations orthogonal to the plane where the magnetic order lies, we take $\mathbf{n}_{i}$ in the $x-y$ plane and we include a spin Jastrow factor $\mathcal{J}_{s}=\exp \left[-\frac{1}{2} \sum_{i, j} u_{i, j} S_{i}^{z} S_{j}^{z}\right]$ in the wave function. ${ }^{24}$ Another density Jastrow factor $\mathcal{J}=\exp \left[-\frac{1}{2} \Sigma_{i, j} v_{i, j} n_{i} n_{j}\right]$ (that includes the on-site Gutzwiller term) is considered to adjust electron correlations. In summary, the correlated wave function is defined by

$$
\left|\Psi_{\mathrm{AF}}\right\rangle=\mathcal{J}_{s} \mathcal{J}|\mathrm{AF}\rangle
$$

Notice that, in this case, the variational state has not a definite total spin, which is suitable for a magnetically ordered phase. In fact, both $|\mathrm{AF}\rangle$ and the spin Jastrow factor $\mathcal{J}_{s}$ break the $\mathrm{SU}(2)$ symmetry.

On the other hand, superconducting or metallic phases can be constructed by considering the ground state $|\mathrm{BCS}\rangle$ of a superconducting Bardeen-Cooper-Schrieffer (BCS) Hamiltonian with both band and pairing contributions ${ }^{25,26}$

$$
\mathcal{H}_{\mathrm{BCS}}=\sum_{q, \sigma} \epsilon_{q} c_{q, \sigma}^{\dagger} c_{q, \sigma}+\sum_{q} \Delta_{q} c_{q, \uparrow}^{\dagger} c_{-q, \downarrow}^{\dagger}+\text { H.c. },
$$

here the band term may also contain a variational chemical potential $\mu$ since the BCS Hamiltonian does not conserve the particle number, i.e., $\epsilon_{q}$ $=-2 t\left(\cos q_{x}+\cos q_{y}\right)-2 t_{d} \cos \left(q_{x}+q_{y}\right)-\mu$. In this case, $t_{d}$ and $\mu$ can be varied to optimize the variational wave function. The full correlated state is given by

$$
\left|\Psi_{\mathrm{BCS}}\right\rangle=\mathcal{J}|\mathrm{BCS}\rangle
$$

in this case, no spin Jastrow is considered, in order to have a perfect singlet state, suitable for a nonmagnetic phase. Notably, within this kind of wave function, it is possible to obtain a pure (i.e., nonmagnetic) Mott insulator just by considering a sufficiently strong Jastrow factor, i.e., $v_{q} \sim 1 / q^{2}\left(v_{q}\right.$ being the Fourier transform of $\left.v_{i, j}\right){ }^{27}$

As we recently demonstrated, ${ }^{14}$ the projected BCS state is not sufficiently accurate for Hubbard-type models, especially in the important strong-coupling regime, i.e., for $U / t \gtrsim 10$, where the superexchange energy scale $J=4 t^{2} / U$ is not correctly reproduced. One efficient way to overcome this problem is to consider backflow correlations ${ }^{14}$ that modify the single-particle orbitals ${ }^{28}$ in the same spirit of what was put forward long time ago by Feynman and Cohen. ${ }^{29}$

Following Ref. 14, we consider a general definition of the new "orbitals" by taking all the possible virtual hoppings of the electrons

$$
\begin{aligned}
\phi_{q}^{b}\left(\mathbf{r}_{i, \sigma}\right) \equiv & \epsilon \phi_{q}\left(\mathbf{r}_{i, \sigma}\right)+\eta_{1} \sum_{j} t_{i j} D_{i} H_{j} \phi_{q}\left(\mathbf{r}_{j, \sigma}\right) \\
& +\eta_{2} \sum_{j} t_{i j} n_{i, \sigma} h_{i,-\sigma} n_{j,-\sigma} h_{j, \sigma} \phi_{q}\left(\mathbf{r}_{j, \sigma}\right) \\
& +\eta_{3} \sum_{j} t_{i j}\left(D_{i} n_{j,-\sigma} h_{j, \sigma}+n_{i, \sigma} h_{i,-\sigma} H_{j}\right) \phi_{q}\left(\mathbf{r}_{j, \sigma}\right),
\end{aligned}
$$

where we used the notation $\phi_{q}\left(\mathbf{r}_{i, \sigma}\right)=\left\langle 0\left|c_{i, \sigma}\right| \phi_{q}\right\rangle$, being $\left|\phi_{q}\right\rangle$ the eigenstates of the mean-field Hamiltonian, $D_{i}=n_{i, \uparrow} n_{i, \downarrow}$, $H_{i}=h_{i, \uparrow} h_{i, \downarrow}$, with $h_{i, \sigma}=\left(1-n_{i, \sigma}\right) . \epsilon, \eta_{1}, \eta_{2}$, and $\eta_{3}$ are variational parameters. As a consequence, already the determinant part of the wave function includes correlation effects. The backflow corrections of Eq. (6) (in particular the $\eta_{1}$ term) make it possible to mimic the effect of the virtual hopping, which leads to the superexchange mechanism. All the parameters of the wave function can be optimized by using the method of Ref. 30.

Finally, the accuracy of the variational calculations can be assessed by using Lanczos diagonalizations on small lattices and Green's function Monte Carlo within the so-called fixednode approximation, ${ }^{31}$ which gives accurate (but approximate) results on large systems. A detailed description of the fixed-node approximation can be found in Ref. 32. In brief, starting from the original Hamiltonian $\mathcal{H}$, we define an effective Hamiltonian by adding a perturbation $O$

$$
\mathcal{H}_{\text {eff }}=\mathcal{H}+O
$$

The operator $O$ is defined through its matrix elements and depends upon a given guiding function $|\Psi\rangle$, that is for instance the variational state itself 
TABLE I. BCS pairing $\Delta$ for various $U / t$ in the metallic region for two sizes of the lattice: $N=100$ (third column) and $N=196$ (fourth column). Notice that for $U / t=8$ and $t^{\prime} / t=0.85$, and for $U / t=6$ and $t^{\prime} / t=0.6$ the BCS wave function is still metallic but the $\mathrm{AF}$ one (insulating) has a lower variational energy.

\begin{tabular}{cccc}
\hline \hline$U / t$ & $t^{\prime} / t$ & $\Delta / t$ & $\Delta / t$ \\
\hline 6 & 0.85 & $0.026(1)$ & $0.018(1)$ \\
7 & 0.85 & $0.051(1)$ & $0.025(1)$ \\
8 & 0.85 & $0.161(1)$ & $0.037(1)$ \\
4 & 0.6 & $0.013(1)$ & $0.005(1)$ \\
5 & 0.6 & $0.027(1)$ & $0.008(1)$ \\
6 & 0.6 & $0.056(1)$ & $0.019(1)$ \\
\hline \hline
\end{tabular}

$$
O_{x^{\prime}, x}= \begin{cases}-\mathcal{H}_{x^{\prime}, x} & \text { if } x^{\prime} \neq x \text { and } s_{x^{\prime}, x}>0 \\ 0 & \text { if } x^{\prime} \neq x \text { and } s_{x^{\prime}, x}<0 \\ \sum_{y: s_{y, x}>0} \mathcal{H}_{y, x} \frac{\Psi_{y}}{\Psi_{x}} & \text { for } x^{\prime}=x,\end{cases}
$$

where $\Psi_{x}=\langle x \mid \Psi\rangle$ and $s_{x^{\prime}, x}=\Psi_{x^{\prime}} \mathcal{H}_{x^{\prime}, x} \Psi_{x}$. Notice that the above operator annihilates the guiding function, namely, $O|\Psi\rangle=0$. Therefore, whenever the guiding function is close to the exact ground state of $\mathcal{H}$, the perturbation $O$ is expected to be small and the effective Hamiltonian becomes very close to the original one.

\section{RESULTS}

By allowing the most general singlet and complex BCS pairing in the state without backflow terms, we find that this quantity has $d_{x^{2}-y^{2}}$ symmetry up to $t^{\prime} \sim t$, namely, the best (nearest-neighbor) pairing function is $\Delta_{q}=2 \Delta\left(\cos q_{x}-\cos q_{y}\right)$, in agreement with previous results. ${ }^{33-36}$ Therefore, within our improved backflow wave function, we only considered a real BCS pairing. We mention that $\Delta$ is very small (especially in the presence of backflow correlations) in the conducting phase, see Table I, and it becomes sizable only in the regime where the magnetic solution prevails over the BCS state. In this regard, we do not find a clear signature of superconductivity close to the metalinsulator transition, as suggested in Ref. 35. We also stress that, once the backflow correlations are considered, there is no energy gain by allowing a translational symmetry breaking (e.g., by considering a $2 \times 1$ unit cell in the BCS Hamiltonian, suitable for dimerized states) and the $d_{x^{2}-y^{2}}$ solution has always a lower energy than dimerized states. Finally, we find that the variational band term of the BCS Hamiltonian $\epsilon_{q}=-2 t\left(\cos q_{x}+\cos q_{y}\right)-2 t_{d} \cos \left(q_{x}+q_{y}\right)-\mu$ has $t_{d} \simeq 0$ for most of the cases considered, except for small $U / t$, inside the conducting phase, where a finite $t_{d}$ can be stabilized.

As far as the magnetic wave function is concerned, both Hartree-Fock and fixed-node calculations give a clear indication that spin-spin correlations remain commensurate at $Q=(\pi, \pi)$ for $t^{\prime} / t \lesssim 0.9$. Therefore, we use an AF wave function having Néel order with pitch vector $Q=(\pi, \pi)$ and we do not consider the implementation of a generic magnetic
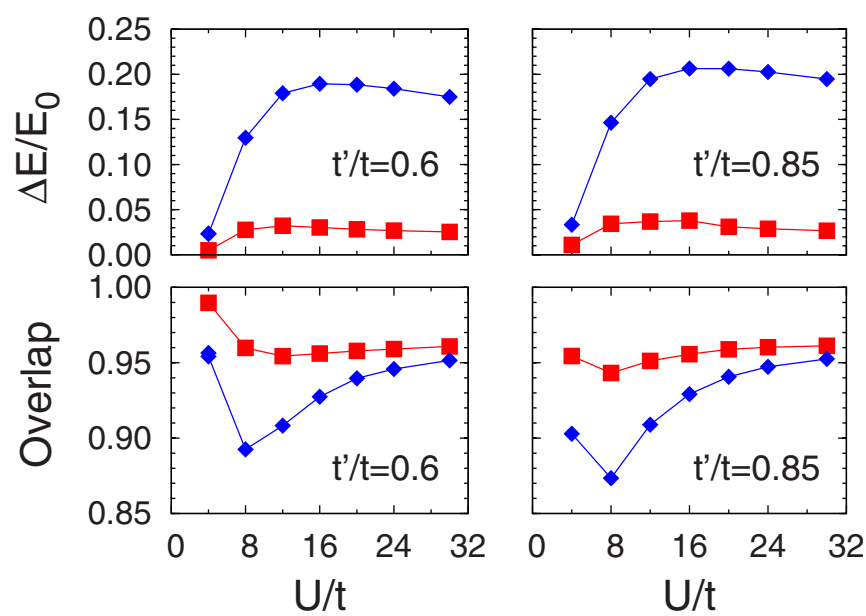

FIG. 2. (Color online) Results for 18 electrons on 18 sites as a function of $U / t$. Upper panels: accuracy of energy $\Delta E=\left(E_{0}-E_{v}\right)$, $E_{v}$ and $E_{0}$ being the variational and the exact values, respectively. Lower panels: overlap between the exact ground state and the variational BCS wave functions. The state without (with) backflow correlations is denoted by diamonds (squares).

state with incommensurate order. Moreover, we verified that, for $t^{\prime} / t \leqq 0.9$, this AF state has a lower energy with respect to the AF state with $120^{\circ}$ order, suitable for $t^{\prime}=t$.

\section{A. Quality of the variational states}

In Fig. 2, we show the accuracy of the BCS variational state and its overlap with the exact ground state in a small lattice with 18 sites (which is tilted by $45^{\circ}$ ). We report two cases with $t^{\prime} / t=0.6$ and 0.85 and different values of $U / t$. As in the case of the frustrated square lattice studied in Ref. 14, the backflow terms highly improve the quality of the variational wave function that remains very accurate even for large correlation, i.e., up to $U / t \sim 30$. We would like to mention that, for this small cluster, the AF state has a slightly lower energy than the BCS one for both $t^{\prime} / t=0.6$ and 0.85 . For $t^{\prime} / t=0.6$, the AF state has also a better overlap with the exact ground state $\left|\Psi_{0}\right\rangle$ (e.g., $\left\langle\Psi_{0} \mid \Psi_{\mathrm{AF}}\right\rangle=0.962$ for $U / t=20$ ) than the BCS state (e.g., $\left\langle\Psi_{0} \mid \Psi_{\mathrm{BCS}}\right\rangle=0.958$ ) while it has a substantially lower overlap for $t^{\prime} / t=0.85$ (e.g., $\left\langle\Psi_{0} \mid \Psi_{\mathrm{AF}}\right\rangle=0.904$ against $\left\langle\Psi_{0} \mid \Psi_{\mathrm{BCS}}\right\rangle=0.959$ ).

The accuracy of the variational state remains very high also for large systems, where the backflow corrections give a sizable and size-consistent improvement. In Fig. 3, we report the energy per site as a function of $U / t$ for both $t^{\prime} / t=0.6$ and 0.85 for $N=10 \times 10$ (see also Table II).

\section{B. Metal-insulator transition}

The metal-insulator transition can be detected by a direct inspection of the static density-density correlations

$$
N_{q}=\frac{1}{N} \sum_{j, l} e^{i q\left(R_{j}-R_{l}\right)}\left\langle n_{j} n_{l}\right\rangle .
$$

In fact, this quantity makes it possible to discriminate between gapless (conducting) and gapped (insulating) phases: a 

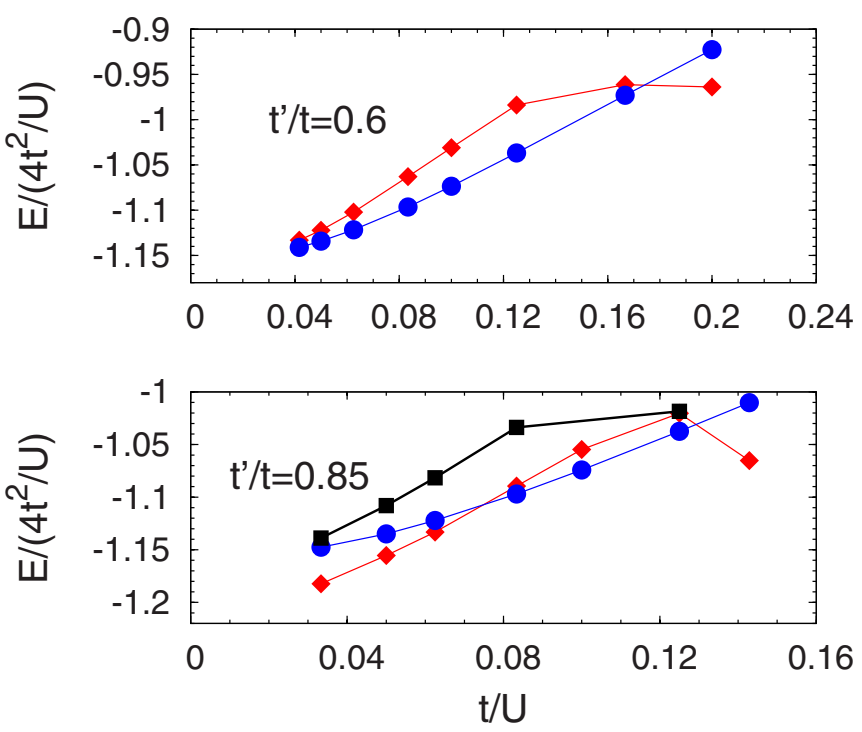

FIG. 3. (Color online) Variational energies per site (in unit of $J=4 t^{2} / U$ ) for the BCS state with a density Jastrow factor (diamonds) and for the AF wave function with both density and spin Jastrow terms (circles); the correlated Fermi gas with Jastrow factor is also reported for $t^{\prime} / t=0.85$ (squares). All states have backflow correlations and results are for 100 sites.

linear behavior $N_{q} \sim|q|$ for $|q| \rightarrow 0$ is typical of a conducting phase, whereas a quadratic behavior $N_{q} \sim q^{2}$ can be associated to an insulating character. ${ }^{27}$ The results presented in Fig. 4 indicate that a metal-insulator transition takes place by increasing $U / t$ and it can be placed at $U_{c}^{\mathrm{MIT}} / t=(5.5 \pm 0.5)$ and $(7.5 \pm 0.5)$ for $t^{\prime} / t=0.6$ and 0.85 , respectively. The transition is first order, with a small jump in the linear coefficient of $N_{q}$ for small momenta. In fact, for small $U / t$, the best wave function is the BCS one (with small superconducting pairing), whereas, by increasing the interaction, the AF one prevails, thus inducing a metal-insulator transition, see Fig. 3.

\section{Insulating phase}

In the insulating regime and for small frustrating ratios, the AF wave function has always a lower energy than the spin-liquid state, and this fact is particularly evident close to

TABLE II. Our best energies per site for $N=100$ : pure variational $E_{\mathrm{vmc}}$ and fixed-node $E_{\mathrm{fn}}$ (still variational) results are reported.

\begin{tabular}{cccc}
\hline \hline$U / t$ & $t^{\prime} / t$ & $E_{\mathrm{vmc}} / t$ & $E_{\mathrm{fn}} / t$ \\
\hline 4 & 0.85 & $-1.03029(2)$ & $-1.0315(1)$ \\
8 & 0.85 & $-0.51876(5)$ & $-0.5238(1)$ \\
12 & 0.85 & $-0.36569(5)$ & $-0.3764(1)$ \\
16 & 0.85 & $-0.2834(1)$ & $-0.2910(1)$ \\
20 & 0.85 & $-0.2311(1)$ & $-0.2364(1)$ \\
4 & 0.6 & $-0.92356(2)$ & $-0.9251(1)$ \\
8 & 0.6 & $-0.51837(3)$ & $-0.5228(1)$ \\
12 & 0.6 & $-0.36550(3)$ & $-0.3689(1)$ \\
16 & 0.6 & $-0.28041(3)$ & $-0.2833(1)$ \\
20 & 0.6 & $-0.22685(3)$ & $-0.2291(1)$ \\
\hline \hline
\end{tabular}
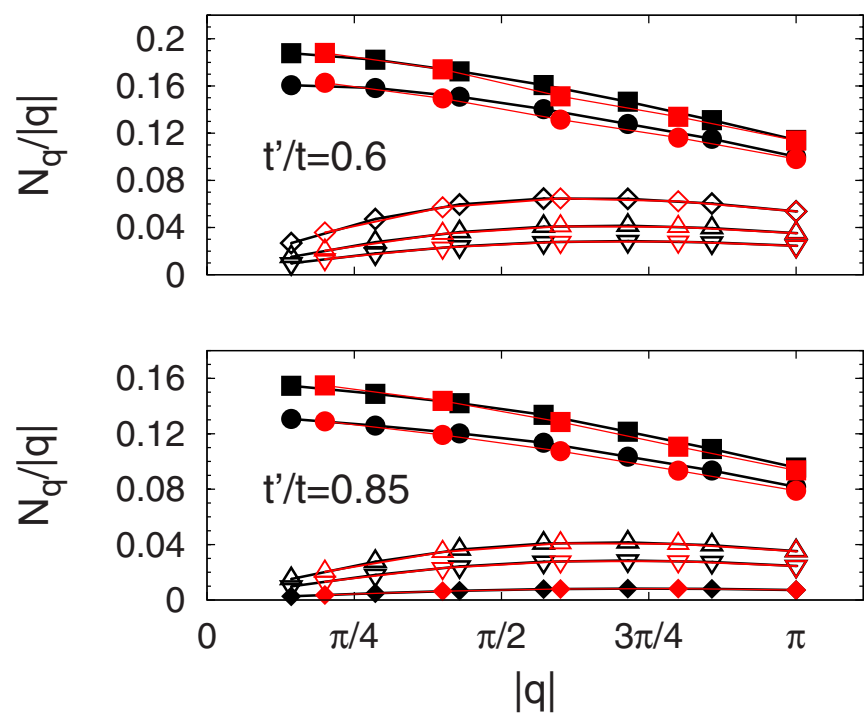

FIG. 4. (Color online) Variational results for the density-density correlations $N_{q}$ divided by $|q|$, along the $(1,0)$ direction, for 100 (red symbols) and 196 (black symbols) sites. Full (empty) symbols refer to the BCS (AF) wave function. Upper panel: from top to bottom, $U / t=4,5,6,8$, and 10. Lower panel: from top to bottom, $U / t=6,7$, 8,10 , and 20 .

the transition, see Fig. 3. On the contrary, for the case with $t^{\prime} / t=0.85$, the BCS state competes with the AF one and it becomes better in energy for $U / t \gtrsim 13$, indicating an insulating spin-liquid behavior at large $U$ (notice that in this region $N_{q} \sim q^{2}$ ). In this regime, the BCS pairing is relevant since the simple projected Fermi sea has a much higher energy, see Fig. 3. Remarkably, the BCS and AF variational energies are always quite close for $t^{\prime} / t=0.85$, suggesting that the actual ground state might be nonmagnetic for all $U>U_{c}^{\mathrm{MIT}}$, or at least down to values lower than expected on the basis of the variational estimate. This fact is supported by the fixed-node calculations for the spin-spin correlations

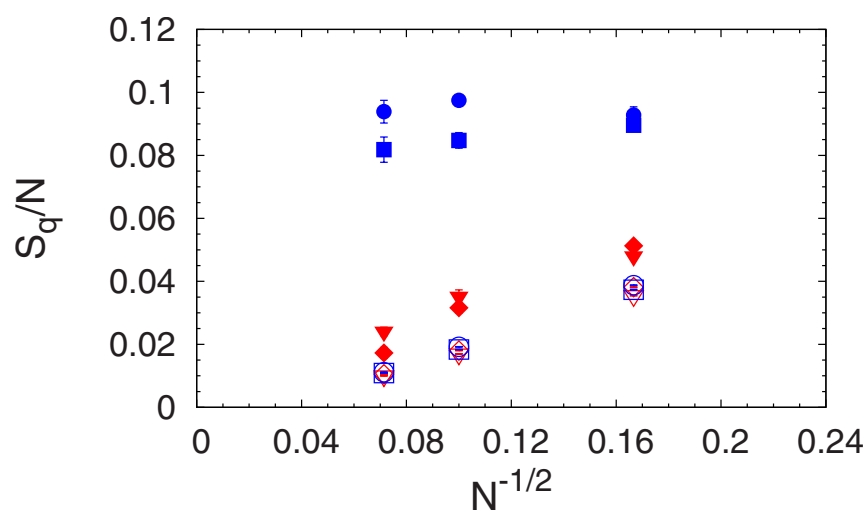

FIG. 5. (Color online) Size scaling of the spin-spin correlations $S_{Q} / N$ for $Q=(\pi, \pi)$. Data are for $t^{\prime} / t=0.6$ with $U / t=10$ (squares) and $U / t=20$ (circles), and $t^{\prime} / t=0.85$ with $U / t=10$ (triangles) and $U / t=20$ (diamonds). Variational and fixed-node results are denoted by empty and full symbols, respectively. The variational results do not depend substantially upon $U$ and $t^{\prime}$. The fixed-node results indicate long-range order for $t^{\prime} / t=0.6$ but not for $t^{\prime} / t=0.85$. 


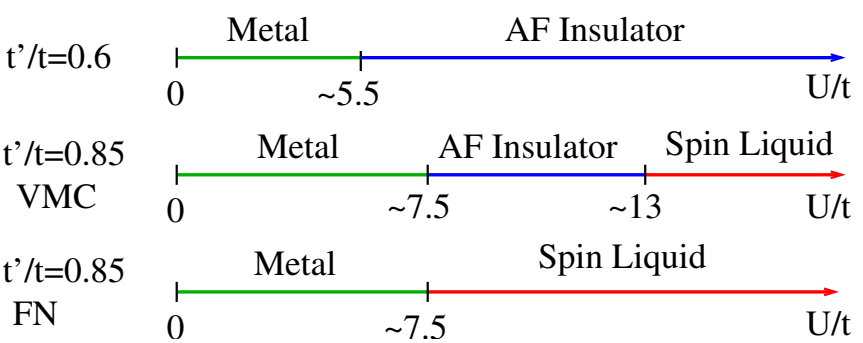

FIG. 6. (Color online) Proposed phase diagram for the two discussed hopping ratios, $t^{\prime} / t=0.6$ and $t^{\prime} / t=0.85$. In the first case, variational $(\mathrm{VMC})$ and fixed-node $(\mathrm{FN})$ results indicate both a direct transition between a metal and an insulator with AF Néel order at a critical value of the electron-electron repulsion $U_{c}$. For $t^{\prime} / t$ $=0.85$, the variational results predict the existence of three different phases at increasing $U / t$ : a metal, an AF insulator with Néel order and a spin liquid, while, within the fixed-node approximation, the nonmagnetic ground state extends down to the metal-insulator transition.

$$
S_{q}=\frac{1}{N} \sum_{j, l} e^{i q\left(R_{j}-R_{l}\right)}\left\langle S_{j}^{z} S_{l}^{z}\right\rangle .
$$

In Fig. 5, we report the size scaling of the variational and the fixed-node results by considering the BCS state as the guiding function. We stress the fact that, in the insulating regime, $S_{q}$ has a peak at the commensurate momentum $Q=(\pi, \pi)$. Remarkably, the fixed-node approach is able to recover a finite value of $S_{Q} / N$ for $Q=(\pi, \pi)$ (i.e., the square of the magnetic order parameter) in the thermodynamic limit for $t^{\prime} / t=0.6$, even though the BCS wave function is nonmagnetic. By contrast, $S_{Q} / N$ tends to zero for $t^{\prime} / t=0.85$ (both for $U / t=10$ and 20), supporting the fact that the ground state is nonmagnetic for this frustrating ratio, even close to the metal-insulator transition. The resulting phase diagram is summarized in Fig. 6.

\section{DISCUSSION}

We have studied the anisotropic triangular lattice at half filling away from the isotropic point $t^{\prime}=t$, with $t^{\prime}<t$, using both a Gutzwiller-Jastrow variational ansatz including backflow correlations as well as a Green's function Monte Carlo approach within the fixed node approximation. We find that the square-lattice states persist up to large values of $t^{\prime} / t<1$, both in terms of the $d$-wave superconducting order parameter as well as for the AF Néel ordering.

The main outcome of this work is that, thanks to the improvement given by backflow correlations, a spin-liquid wave function can be stabilized over magnetic states, for large but still moderate Coulomb repulsions and close to the isotropic limit. These variational results are corroborated by fixed-node calculations. We find, in particular, that for $t^{\prime} / t$ $=0.85$, which is relevant for $\kappa-(\mathrm{ET})_{2} \mathrm{Cu}_{2}(\mathrm{CN})_{3},{ }^{12,13}$ the insulating phase has a pure Mott character, without magnetic order. On the other hand, for $t^{\prime} / t=0.6$, suitable for $\kappa$ - $(\mathrm{ET})_{2} \mathrm{Cu}_{2}(\mathrm{SCN})_{2}$, (or even smaller $t^{\prime} / t$ values) the insulating phase always shows Néel order with $Q=(\pi, \pi)$.
Let us finish by discussing our results also in comparison to other calculations and experimental findings. First of all, in various papers, it has been suggested that the spin-liquid phase can be stabilized by charge fluctuations that may destabilize a magnetically ordered state. This claim has been corroborated by calculations on Heisenberg models in presence of a ring-exchange term $J_{4}$ (that appears in the strongcoupling expansion in $\left.t^{4} / U^{3}\right) .{ }^{37}$ However, it turns out that the actual value of $J_{4}$ for stabilizing a disordered phase is rather large and, probably, beyond the validity of a perturbative expansion. The existence of a direct transition from a magnetic phase to a disordered one has also been found in the original Hubbard model, by decreasing the on-site repulsion $U .{ }^{16,17,21}$ We do not find any evidence of such a possibility and, in our approach, the magnetic phase is stable in presence of charge fluctuations, even close to the metal-insulator transition: this is the case of $t^{\prime} / t=0.6$. Instead, the spin-liquid phase turns out to be directly connected with the one at strong coupling while antiferromagnetic correlations become stronger when decreasing $U / t$. For example, for $t^{\prime} / t=0.85$, the variational state with magnetic order has a slightly lower energy close to $U_{c}$ and we need to apply the Green's function Monte Carlo approach to extend the spin-liquid region down to the metal-insulator transition, see Fig. 6. At this stage, we would also like to mention that the metallic phase is likely to be not superconducting. In fact, the BCS pairing $\Delta$ in the metallic region is slightly suppressed when improving the accuracy of the variational wave function by considering backflow correlations and, moreover, it is reduced by a factor 2-3 when the lattice size is increased from $10 \times 10$ to $14 \times 14$, see Table I. This fact contrasts the previous claim of a possible superconducting phase close to the metal-insulator transition by Liu and collaborators. ${ }^{35}$

Another very important point is to clarify the nature of the low-energy excitations. Very recently, thermodynamic measurements of the specific heat suggested the possible existence of a Fermi surface of neutral, $S=1 / 2$ fermionic spinons. ${ }^{38}$ However, it should be noticed that such a measurement involves a difficult subtraction of a divergent nuclear specific heat and instead the thermal conductivity (which is not affected by a nuclear contribution) shows an activated behavior with a tiny gap of $0.46 \mathrm{~K} .{ }^{39}$ This fact has been associated with the existence of spinless "vison" excitations. ${ }^{40}$ From our calculations, it appears that the disordered insulating phase cannot sustain a true spinon Fermi surface, as previously suggested both on variational calculations ${ }^{37}$ and field-theory approaches, ${ }^{41}$ but it has Dirac points at $( \pm \pi / 2, \pm \pi / 2)$. In fact, the projected Fermi sea has a much higher energy than our best variational ansatz with BCS pairing, see Fig. 3. Should our results be correct, either a deeper investigation of the minimal microscopic model for describing organic charge-transfer salts is needed, or a reinterpretation of the experimental data is required.

\section{ACKNOWLEDGMENTS}

We thank R. Valenti for very useful discussions. L.T. and C.G. thank partial support from the German Science Foundation through the Transregio 49. 
${ }^{1}$ J. Solyom, Adv. Phys. 28, 201 (1979).

${ }^{2}$ K. Kanoda, J. Phys. Soc. Jpn. 75, 051007 (2006).

${ }^{3}$ K. Miyagawa, A. Kawamoto, Y. Nakazawa, and K. Kanoda, Phys. Rev. Lett. 75, 1174 (1995).

${ }^{4}$ H. Elsinger, J. Wosnitza, S. Wanka, J. Hagel, D. Schweitzer, and W. Strunz, Phys. Rev. Lett. 84, 6098 (2000).

${ }^{5}$ S. Lefebvre, P. Wzietek, S. Brown, C. Bourbonnais, D. Jerome, C. Meziere, M. Fourmigue, and P. Batail, Phys. Rev. Lett. 85, 5420 (2000).

${ }^{6}$ P. Limelette, P. Wzietek, S. Florens, A. Georges, T. A. Costi, C. Pasquier, D. Jerome, C. Meziere, and P. Batail, Phys. Rev. Lett. 91, 016401 (2003).

${ }^{7}$ F. Kagawa, T. Itou, K. Miyagawa, and K. Kanoda, Phys. Rev. B 69, 064511 (2004).

${ }^{8}$ Y. Kurosaki, Y. Shimizu, K. Miyagawa, K. Kanoda, and G. Saito, Phys. Rev. Lett. 95, 177001 (2005).

${ }^{9}$ Y. Shimizu, K. Miyagawa, K. Kanoda, M. Maesato, and G. Saito, Phys. Rev. Lett. 91, 107001 (2003).

${ }^{10}$ H. Kino and H. Fukuyama, J. Phys. Soc. Jpn. 65, 2158 (1996).

${ }^{11}$ R. H. McKenzie, Comments Condens. Matter Phys. 18, 309 (1998); Science 278, 820 (1997).

${ }^{12}$ K. Nakamura, Y. Yoshimoto, T. Kosugi, R. Arita, and M. Imada, J. Phys. Soc. Jpn. 78, 083710 (2009).

${ }^{13}$ H. C. Kandpal, I. Opahle, Y.-Z. Zhang, H. O. Jeschke, and R. Valentí, Phys. Rev. Lett. 103, 067004 (2009).

${ }^{14}$ L. F. Tocchio, F. Becca, A. Parola, and S. Sorella, Phys. Rev. B 78, 041101(R) (2008).

${ }^{15}$ At half filling, the sign of $t^{\prime}$ is irrelevant.

${ }^{16}$ R. T. Clay, H. Li, and S. Mazumdar, Phys. Rev. Lett. 101, 166403 (2008).

${ }^{17}$ H. Morita, S. Watanabe, and M. Imada, J. Phys. Soc. Jpn. 71, 2109 (2002).

${ }^{18}$ T. Watanabe, H. Yokoyama, Y. Tanaka, and J. Inoue, J. Phys. Soc. Jpn. 75, 074707 (2006).

${ }^{19}$ T. Watanabe, H. Yokoyama, Y. Tanaka, and J. Inoue, Phys. Rev. B 77, 214505 (2008).

${ }^{20}$ S. Guertler, Q.-H. Wang, and F. C. Zhang, Phys. Rev. B 79, 144526 (2009).

${ }^{21}$ B. Kyung and A.-M. S. Tremblay, Phys. Rev. Lett. 97, 046402
(2006).

${ }^{22}$ T. Ohashi, T. Momoi, H. Tsunetsugu, and N. Kawakami, Phys. Rev. Lett. 100, 076402 (2008).

${ }^{23}$ H. Lee, G. Li, and H. Monien, Phys. Rev. B 78, 205117 (2008).

${ }^{24}$ F. Becca, M. Capone, and S. Sorella, Phys. Rev. B 62, 12700 (2000).

${ }^{25}$ C. Gros, Phys. Rev. B 38, 931(R) (1988).

${ }^{26}$ F. C. Zhang, C. Gros, T. M. Rice, and H. Shiba, Supercond. Sci. Technol. 1, 36 (1988).

${ }^{27}$ M. Capello, F. Becca, M. Fabrizio, S. Sorella, and E. Tosatti, Phys. Rev. Lett. 94, 026406 (2005).

${ }^{28}$ Single-particle states are defined in the BCS Hamiltonian, after the transformation $c_{i, \downarrow}^{\dagger} \rightarrow c_{i, \downarrow}$.

${ }^{29}$ R. P. Feynman and M. Cohen, Phys. Rev. 102, 1189 (1956).

${ }^{30}$ S. Yunoki and S. Sorella, Phys. Rev. B 74, 014408 (2006).

${ }^{31}$ D. F. B. ten Haaf, H. J. M. van Bemmel, J. M. J. van Leeuwen, W. van Saarloos, and D. M. Ceperley, Phys. Rev. B 51, 13039 (1995).

${ }^{32}$ M. Lugas, L. Spanu, F. Becca, and S. Sorella, Phys. Rev. B 74, 165122 (2006).

${ }^{33}$ B. J. Powell and R. H. McKenzie, Phys. Rev. Lett. 94, 047004 (2005).

${ }^{34}$ J. Y. Gan, Y. Chen, Z. B. Su, and F. C. Zhang, Phys. Rev. Lett. 94, 067005 (2005).

${ }^{35}$ J. Liu, J. Schmalian, and N. Trivedi, Phys. Rev. Lett. 94, 127003 (2005).

${ }^{36}$ B. J. Powell and R. H. McKenzie, Phys. Rev. Lett. 98, 027005 (2007).

${ }^{37}$ O. I. Motrunich, Phys. Rev. B 72, 045105 (2005).

${ }^{38}$ S. Yamashita, Y. Nakazawa, M. Oguni, Y. Oshima, H. Nojiri, Y. Shimizu, K. Miyagawa, and K. Kanoda, Nat. Phys. 4, 459 (2008).

${ }^{39}$ M. Yamashita, N. Nakata, Y. Kasahara, T. Sasaki, N. Yoneyama, N. Kobayashi, S. Fujimoto, T. Shibauchi, and Y. Matsuda, Nat. Phys. 5, 44 (2009).

${ }^{40}$ Y. Qi, C. Xu, and S. Sachdev, Phys. Rev. Lett. 102, 176401 (2009).

${ }^{41}$ S.-S. Lee, P. A. Lee, and T. Senthil, Phys. Rev. Lett. 98, 067006 (2007). 